\title{
Best Fit based VM Allocation for Cloud Resource Allocation
}

\author{
Saurabh Shirvastava \\ LNCT, Bhopal
}

\author{
Rahul Dubey \\ LNCT, Bhopal
}

\author{
Manish Shrivastava \\ LNCT, Bhopal
}

\begin{abstract}
Cloud consists of datacenters with each datacenter having large number of physical machines. On top of each physical machine a virtual machine is created. In this paper a clustering based solution for VM migrations and consolidation is proposed. The proposed best fit VM placement approach is also energy efficient as compared to traditional VM placement approach.
\end{abstract}

\section{Keywords}

computing, VM placement and consolidation, Best fit approach.

\section{INTRODUCTION}

Cloud computing could be a model for providing service as Platform, Software, Hardware as a service over web facultative present, convenient, on-demand network access to a shared pool of configurable computing resources (e.g., networks, servers, storage, applications, and services) that may be speedily provisioned and discharged with strippeddown management effort or service supplier interaction [1]. Cloud computing is that the latest effort in delivering computing resources as a service. User will rent computing resources on-line as a product these computing resources are going to be utilized by user on-line [2], this helps in reducing prices offer|and supply\}rs can take hold of it and provide on demand service that's delivered to shoppers over the web from large-scale data centres or "clouds". while cloud computing is gaining growing popularity within the IT trade. Cloud Computing encompass assortment of distributed servers called masters who give demanded services and resources to the purchasers called central controller or cloud manager in a very network with the quantifiability and responsibility of servers. On-demand service are going to be provided by distributed servers. Services could also be of software resources (e.g. software as a Service, SaaS)[3] or physical resources (e.g. Platform as a Service, PaaS) or hardware/infrastructure (e.g. Hardware as a Service, HaaS [4] or Infrastructure as a Service, IaaS). Amazon EC2 (Amazon Elastic compute Cloud) is associate example of cloud computing services. Cloud services are provisioned to use by service providers, for example, Amazon, Google on the Internet. Usually, the resources available to the user of the cloud are virtualized that is (Paas, Iaas, and Saas) services are virtual service. User gets required service without any dependencies or constraints in return companies will take some charge for using their services which are nominal as compared to the actual cost of that particular service, because of this cloud is becoming popular.

Cloud computing technology uses web and central remote servers to take care of data and applications [11]. Cloud computing permits any user from anyplace to use the updated version of services and application. we need to not purchase software system with the license as a result of change and maintaining software system are the server's responsibility, solely we want to possess web connection, thereupon we are able to use applications while not putting in it on our system. Gmail, yahoo mail or Hotmail, etc. are the common and wide used cloud example, for mistreatment any quite cloud service, you need to have an online affiliation. Scientific application wants an outsized quantity of calculation and storage that you wants an outsized computation storage and power. Initially, all the scientific applications are deployed on Grid [5]. However, Grid computing is expensive and not offered everywhere the word. so the scientific applications are moving toward the cloud, Cloud provides an alternate to grid and supercomputers for a individual in a very lower price. Cloud is an rising space and ideal for this type of application. For the development of deploying an application of cloud there are such a lot of methods developed, for instance load equalization, planning algorithmic program for $\mathrm{VM}$ allocation in cloud.

Virtualization: Virtualization may be a layer between Hardware and software system. at the start mainframe is employed to support several users victimization the virtual machine terminal. This terminal shows the simulated behavior of an software system for every user. VMware workstation may be a similar product started in 1999 and it facilitates to run multiple operational systems in personal computers. Virtualization is that the foundation of cloud technology. Users will access resources for computing or for storage victimization virtualization while not knowing background detail [6].

Virtual Machine Allocation: VM allocation permits economical sharing of virtual machines to obtainable data centres and these allocation strategies facilitate to guage and enhance the performance of cloud [7]. completely different allocation policies are obtainable and that they have their own benefits and limitations. the most important objective of each VM allocation methodology is to reduce time. Throttled Load Balancer (TLB) [9] is additionally the similar analysis done before.

\section{PROPOSED APPROACH}

When cloud received a request, it first needs to select the appropriate cluster for the deployment of cloud application, by selecting a Cluster, which consists of two or more VM and other resources; it will select the freely available VM, and will allocate the job to the selected VM. The proposed Algorithm uses an index approach for VM selection. In this approach the indexing is done to all the resources with their status as it is available or not in this way it can be easily selected in a fast manner. It is explained in the following steps.

Algorithm VM Allocation
$\left\{\begin{array}{l}\text { STEP } 1 \text { - The cloud manager initially forms the Indexes of } \\ \text { each CLUSTER, Indexes of each VM within each }\end{array}\right.$


CLUSTER, and Indexes of each resource available within each VM.

STEP 2 - The cloud manager collects the Indexes of current VMs in use and indexes of CLUSTER in which currently active $\mathrm{VM}$ is present.

STEP 3 - Cloud manager generate a reference table to store the status of different VMs and CLUSTERs for example, let the selected CLUSTER is number 4, selected VM within CLUSTER is 3 and all other CLUSTER and VM remains idle, then it will be represented.

STEP 4 - One extra column is added in reference table which shows the status of that CLUSTER whether it is started or not in case of CLUSTER.

' 0 ' represents CLUSTER is off.

' 1 ' represents CLUSTER is started.

In case of VMs,

' 0 ' represents that VM is free.

' 1 ' represents that VM already has data.

Whenever any CLUSTER is selected for the first time, its entry is made in the reference table along with its CLUSTER and all the VM within it, and the table is immediately updated after any action is performed on VM or CLUSTER level either assigning workload (wakeup) or turning off (power down) it.

Initially the entire CLUSTER is in off state and all the VM within them remain started.

This is done in order to assure that whenever a new cluster is selected that was in off state, only one wakeup signal will be sufficient to wakeup whole CLUSTER for reducing further latency to wake up each VM.

\section{EXPERIMENTAL SETUP \& RESULTS}

In this research a cloud is simulated using Cloudsim having fixed number of physical machines and virtual machines. Configuration of physical and virtual machines is measured in terms of MIPS (million instructions per second). VM Migrations, VM consolidation and energy consumption are recorded.

Physical machines are created based on the following MIPS list as shown below:

$$
\{750,1000,1500,2000\}
$$

Virtual machines are created based on the following MIPS list as shown below:

$$
\{250,500,750,1000\}
$$

No. of VM Migrations are compared for traditional VM Migration approach (TVMM), and Best Fit based approach (BFVMM).

Table 1: Table showing number of VM migrations for both approaches

\begin{tabular}{|c|c|c|c|}
\hline $\begin{array}{c}\text { No. of } \\
\text { PMs }\end{array}$ & $\begin{array}{c}\text { No. of } \\
\text { VMs }\end{array}$ & TVMM & BFVMM \\
\hline 10 & 15 & 7 & 1 \\
\hline 15 & 20 & 9 & 2 \\
\hline 20 & 25 & 11 & 6 \\
\hline 25 & 30 & 16 & 10 \\
\hline 30 & 35 & 19 & 11 \\
\hline
\end{tabular}

The above table clearly shows that number of VM migrations increase with the increase in number of VM. In traditional VM migration (TVMM) number of VM migration is more as compared to base approach Best Fit VM migration.

Table 2: Table showing number of VM cpnsolidations for both approaches

\begin{tabular}{|c|c|c|c|}
\hline $\begin{array}{c}\text { No. of } \\
\text { PMs }\end{array}$ & $\begin{array}{c}\text { No. of } \\
\text { VMs }\end{array}$ & TVMM & BFVMM \\
\hline 10 & 15 & 2 & 7 \\
\hline 15 & 20 & 3 & 6 \\
\hline 20 & 25 & 5 & 10 \\
\hline 25 & 30 & 8 & 14 \\
\hline 30 & 35 & 11 & 18 \\
\hline
\end{tabular}

VM consolidation means number of PMs having less utilization switched to sleep mode by migrating all its VMs to other VMs having normal utilization. Number of VM consolidated should be more. And in proposed approach number of VM consolidated are more.

Table 1 : Table showing energy consumptions in KWH for both approaches

\begin{tabular}{|c|c|c|c|}
\hline $\begin{array}{c}\text { No. of } \\
\text { PMs }\end{array}$ & $\begin{array}{c}\text { No. of } \\
\text { VMs }\end{array}$ & TVMM & BFVMM \\
\hline 10 & 15 & 3 & 1 \\
\hline 15 & 20 & 5 & 2 \\
\hline 20 & 25 & 7 & 6 \\
\hline 25 & 30 & 8 & 7 \\
\hline 30 & 35 & 12 & 10 \\
\hline
\end{tabular}

Energy consumption should be as minimum as possible. And in proposed approach energy consumption is also minimum.

\section{CONCLUSION}

In this paper a energy efficient scheme for VM placement is proposed based on clustering. Clustering is done to group the servers into categories based on whether they have VMs running on it or not. Clustering is done to switch off the servers which do not have any VMs running on it. The proposed scheme is compared with the traditional approach for VM placement and consolidation. It is observed experimentally that proposed best fit approach is better in terms of VM migrations, VM consolidations and energy consumption.

\section{REFERENCES}

[1] http://www.wikinvest.com/concept/Cloud_Computing.

[2] Mladen A. Vouk, Cloud Computing - Issues, Research and Implementations, Information technology Interfaces $30^{\text {th }}$ International conference, Vol. 4, pp. 235-246, June 2008.

[3] Pei Fan and Zhenbang Chen and JiWang, TopologyAware Deployment of Scientific Applications in cloud computing, Int. J. Web and Grid Services, Vol. 36, PP. 319 - 326, July 2014

[4] Bhupendra Panchal and Prof. R. K. Kapoor, "Dynamic VM Allocation Algorithm using Clustering in Cloud Computing", International Journal of advanced research 
in computer science and software engineering, Volume 3, Issue 9, PP. 143-150, September 2011.

[5] Daniel A. Menasc'e Paul Ngo, "Understanding Cloud Computing: Experimentation and Capacity Planning", Proc. Computer Measurement Group Conf, Dallas, TX, Dec. 7-11, 2009.

[6] Calheiros R.N., "Virtual Machine Provisioning Based on Analytical Performance and QoS in Cloud Computing Environments", International Conference on Parallel Processing, page no 295-304,Sept 2011.

[7] Michael Shindler, Alex Wong, "Fast and Accurate kmeans For Large Datasets", PP. 1-9, 2011.

[8] Soumya Ray and Ajanta De Sarkar, "Execution analysis of load balancing algorithm in cloud computing environment", International Journal on Cloud Computing: Services and Architecture (IJCCSA), Vol.2, No.5, PP. 1-13, October 2012.

[9] Ahmed ali eldin, Maria Kihl and Johan Tordsson, "Efficient provisioning of bursty scientific workload on the Cloud Using Adaptive Elasticity Contro"l, journal of
Computer Science Issues, Vol. 9, Issue 1, No 1, PP. 3140, January 2012.

[10] Nidhi Jain Kansal, Inderveer Chana, "Cloud Load Balancing Techniques : A Step Towards Green Computing, International Journal of Computer Science Issues, Vol. 9, Issue 1, No 1, PP. 1694-0814, January 2012.

[11] Rajleen Kaur, Amanpreet Kaur, "A Review Paper on Evolution of Cloud Computing, its Approaches and Comparison with Grid Computing", International Journal of Computer Science and Information Technologies, Vol. 5, PP. 6060-6063, 2014.

[12] Abhishek Gupta, Osman Sarood, Laxmikant V Kale, "Optimizing VM Placement for HPC in the Cloud", International Letters of Social and Humanistic Sciences, Vol. 16, PP. 1-6, 2014.

[13] Dzmitry Kliazovich, Sisay T. Arzo, Fabrizio Granelli, "e-STAB: Energy-Efficient Scheduling for Cloud Computing Applications with Traffic Load Balancing", IEEE International Conference on Green Computing and Communications, pp. 7-13, 2013. 